1 Departamento de Nutrição, Faculdade de Saúde Pública, Universidade de São Paulo (FSPUSP), São Paulo, SP, Brasil

\author{
Correspondência para: \\ Sandra Roberta G. Ferreira \\ Departamento de Nutrição, \\ Faculdade de Saúde Pública, \\ Universidade de São Paulo \\ Av. Dr. Arnaldo, 715 \\ 01246-904 - São Paulo, SP, Brasil \\ sandrafv@usp.br
}

Recebido em 29/Jan/2010 Aceito em 1/Ago/2010

\section{Análise de diferentes medidas antropométricas na identificação de síndrome metabólica, com ou sem alteração do metabolismo glicídico}

\author{
Analysis of several anthropometric measurements for the identification of \\ metabolic syndrome, with or without disturbance of glucose metabolism
}

Natália Pereira da Rocha' , Antonela Siqueira-Catania', Camila Risso Barros' ‘ Milena Monfort Pires' ${ }^{1}$ Luciana D. Folchetti', Sandra Roberta G. Ferreira'

\section{RESUMO}

Objetivo: Este estudo comparou parâmetros antropométricos e de resistência à insulina de indivíduos sem e com síndrome metabólica (SM), subestratificados pela presença de anormalidades glicêmicas. Sujeitos e métodos: Foram incluídos 454 indivíduos (66\% mulheres, $54 \%$ brancos), sendo 155 alocados para o grupo 1 (sem SM, sem anormalidade glicêmica), 32 para o grupo 2 (sem SM, com anormalidade glicêmica), 104 no grupo 3 (com SM, sem anormalidade glicêmica) e 163 no grupo 4 (com SM e anormalidade glicêmica). Os grupos foram comparados por ANOVA. Resultados: Os grupos com SM (3 e 4) apresentaram os piores perfis antropométrico e lipídico; no grupo 2, apesar de glicemias significantemente mais elevadas, as médias das variáveis antropométricas e lipídicas não diferiram do grupo 1. Os maiores valores médios de HOMA-IR foram encontrados nos grupos com SM, enquanto o grupo 2 apresentou o menor HOMA- $\beta$. A trigliceridemia foi a variável metabólica com coeficientes de correlação mais elevados com a antropometria. Porém, as correlações mais fortes foram da circunferência da cintura $(r=0,503)$ e da razão cinturaaltura $(r=0,513)$ com o HOMA-IR $(p<0,01)$. Conclusão: Nossos achados revelam que, em amostra da população brasileira, qualquer das medidas antropométricas identifica indivíduos com SM, mas não parece capaz de diferenciar aqueles com distúrbio glicêmico. Reforçamos a relação mais forte das medidas de adiposidade central com resistência à insulina, sugerindo utilidade da razão cintura-altura. É possível que componente autoimune contribua para o comprometimento do metabolismo glicídico dos indivíduos do grupo 2. Arq Bras Endocrinol Metab. 2010;54(7):636-43

\section{Descritores}

Antropometria; índice de massa corporal; circunferência abdominal; razão cintura-altura; síndrome metabólica; distúrbios glicêmicos

\section{ABSTRACT}

Objective: This study compared anthropometric measurements and insulin resistance indexes of individuals with or without metabolic syndrome (MS), stratified by the presence of glycemic abnormalities. Subjects and methods: 454 individuals (66\% women, $54 \%$ Caucasians) were included, being 155 allocated to group 1 (without MS, without glycemic abnormality), 32 to group 2 (without MS, with glycemic abnormality), 104 to group 3 (with MS, without glycemic abnormality), and 163 to group 4 (with MS, with glycemic abnormality). Groups were compared by ANOVA. Results: Those with MS (3 e 4) showed the worst anthropometric and lipid profiles; in group 2, despite higher plasma glucose levels, the mean values of anthropometric variables and lipids did not differ from group 1. The highest mean values of HOMA-IR were found in the groups with MS, while group 2 showed the lowest HOMA- $\beta$. Triglyceride was the metabolic variable with the highest correlation coefficients with anthropometry. However, the strongest correlations were those of waist circumference $(r=$ $0.503)$ and waist-to-height ratio $(r=0.513)$ with HOMA-IR $(p<0.01)$. Conclusion: Our findings indicate that, in a sample of the Brazilian population, any anthropometric measure identifies individuals with MS, but such measurements seem to be unable to differentiate those with glycemic disturbance. We reinforce the strongest relationship of measures of central adiposity with insulin resistance, suggesting utility for the waist-to-height. An autoimmune component may be contributing to the deterioration of glucose metabolism of individuals from group 2. Arq Bras Endocrinol Metab. 2010;54(7):636-43

\section{Keywords}

Anthropometry; body mass index; waist-to-height ratio; metabolic syndrome; glycemic disturbances 


\section{INTRODUÇÃO}

$\mathrm{P}$ revalências crescentes de obesidade são observadas nos países desenvolvidos e também nos países em desenvolvimento como o Brasil (1-3). Particularmente o acúmulo abdominal da gordura corporal prediz distúrbios metabólicos que compõem a síndrome metabólica (SM), cujo mecanismo fisiopatológico central é a resistência à insulina (4-6). Ao espectro das anormalidades da SM pertence um estado inflamatório subclínico $(7,8)$, comumente avaliado pela determinação da concentração sérica de proteína $\mathrm{C}$ reativa. Esta se encontra aumentada na obesidade (9), sendo preditiva de evento cardiovascular (10). A importância do diagnóstico de SM baseia-se no risco de progressão ao diabetes melito (DM) tipo $2(11,12)$ e de eventos cardiovasculares (1315). O DM per se é indicativo de alto risco cardiovascular, uma vez que o risco de infarto do miocárdio no indivíduo diabético é similar ao daquele não diabético que infartou anteriormente (16). A presença de DM na SM potencializa o risco cardiovascular (17).

Condições pré-diabéticas, glicemia de jejum (GJA) ou tolerância à glicose diminuída (TGD) também se associam a morbimortalidade cardiovascular (18), havendo indicação de tratamento para minimizar riscos. Nesta etapa da história natural do DM tipo 2, a resistência à insulina e a inflamação subclínica já estão presentes (19).

Medidas antropométricas são frequentemente empregadas na avaliação da adiposidade corporal devido à sua praticidade e baixo custo. $\mathrm{O}$ índice de massa corporal (IMC) é a medida mais utilizada e estudos epidemiológicos mostram sua clara associação com elevação de mortalidade $(20,21)$. Medidas regionais de obesidade, entre as quais a medida da circunferência da cintura (CC) e a razão cintura/quadril (RCQ), são capazes de estimar indiretamente a gordura intra-abdominal que reflete a massa de tecido adiposo visceral, gerador de resistência à insulina. Essas medidas se mostram preditivas de distúrbios metabólicos, doença cardiovascular e morte (22-24). A circunferência da cintura é considerada o principal parâmetro diagnóstico da SM pela International Diabetes Federation - IDF (25). Para populações latinas ou miscigenadas ainda não estão disponíveis estudos epidemiológicos apontando a melhor medida antropométrica para esse fim ou seu ponto de corte.

Recomenda-se obtenção de medida da centralidade da gordura corporal na rotina clínica que, combinada ao IMC, complementa a capacidade preditiva de com- plicações cardiovasculares $(25,26)$. Foi proposto que a razão cintura/altura (RCA) seria bom índice antropométrico na predição de DM, risco cardiovascular e de SM (27-30). Porém, são escassos estudos em estratos da população brasileira, caracterizada por alta miscigenação étnica. Esse fato dificulta estabelecer valores de corte para variáveis antropométricas na predição de risco. A vantagem da RCA nesse sentido é que o ajuste pela estatura tende a adequar a medida da circunferência da cintura para indivíduos de diferentes etnias. Estudo recente avaliou a capacidade de identificar distúrbios metabólicos por meio de medidas antropométricas numa amostra multiétnica (30).

A avaliação da composição corporal pode ser complementada por métodos especializados, capazes de fornecer estimativas precisas, porém invasivos e de alto custo (31). Não é o caso da bioimpedância elétrica, que fornece os percentuais de gordura total e massa magra, sendo de pouca valia na estimativa específica da gordura intra-abdominal.

É possível que o perfil antropométrico de portadores ou não de SM sem alteração do metabolismo glicídico seja menos desfavorável quando comparado àqueles que já desenvolveram anormalidades da tolerância à glicose. Supõe-se que parâmetros antropométricos indicativos de centralidade da gordura corporal - sugestivos de resistência à insulina - sejam capazes de identificar portadores de distúrbios glicêmicos. O objetivo deste estudo foi comparar diversos parâmetros antropométricos e de resistência à insulina entre indivíduos sem e com SM, subestratificados pela presença de anormalidades do metabolismo glicídico. Também foi examinada a correlação entre variáveis antropométricas com índice de resistência à insulina.

\section{SUJEITOS E MÉTODOS}

\section{Pacientes}

Os participantes do presente estudo foram obtidos a partir do rastreamento de indivíduos para integrar um programa de intervenção no estilo de vida (hábitos alimentares e atividade física) para prevenção de DM tipo 2, em andamento no Centro de Saúde da Faculdade de Saúde Pública da Universidade de São Paulo (FSPUSP). As fontes desses indivíduos foram o Inquérito de Saúde do Adulto - ISA-Capital (ㄹef) e, para completar a amostra do referido programa, foram rastreados funcionários da Universidade e usuários do Centro. Os crité- 
rios de inclusão para o presente estudo foram indivíduos de ambos os sexos, com idade $\geq 18$ anos, preenchendo ou não critérios diagnósticos para SM (32). Aqueles rastreados sem distúrbios metabólicos compuseram um "grupo controle". Foram excluídos indivíduos em uso de medicamentos antiobesidade e gestantes. Este estudo foi aprovado pelo Comitê de Ética institucional e os participantes assinaram termo de consentimento após terem sido esclarecidos sobre seus propósitos.

\section{Métodos}

Trata-se de uma análise transversal do banco de dados de indivíduos rastreados, previamente à intervenção a que seriam submetidos. A avaliação dos participantes compreendeu, além de dados demográficos, obtenção de medidas antropométricas, pressão arterial e de bioimpedância elétrica. Também foram submetidos à coleta de sangue em jejum para determinações bioquímicas e ao teste oral de tolerância à glicose (TOTG) com $75 \mathrm{~g}$, segundo recomendações da Organização Mundial da Saúde (OMS).

A antropometria foi realizada por examinadores treinados. Foram obtidos peso, com o mínimo de roupa e sem sapatos, em balança eletrônica, e a altura por estadiômetro. Para cálculo do IMC, o peso $(\mathrm{kg})$ foi dividido pela altura $(\mathrm{m})$ ao quadrado. As circunferências da cintura (meia distância entre a última costela flutuante e a crista ilíaca) e do quadril foram obtidas em pé, por meio de fita métrica inelástica, usando técnica preconizada pela OMS (33). Foram obtidas a RCQ por meio do quociente da circunferência da cintura pela do quadril, e a RCA dividindo-se a circunferência da cintura pela estatura. Considerou-se como limite da normalidade da RCA o valor de 0,50 . A recomendação de manter a circunferência da cintura abaixo da metade da altura foi previamente publicada (34).

Estimativas da massa gorda e massa magra foram obtidas por meio do aparelho de bioimpedância RJL, modelo Quantum II e software específico.

Três medidas de pressão arterial foram obtidas com esfigmomanômetro automático (Omron model HEM712C, Omron Health Care, Inc, USA), na posição sentada, após 5 minutos de repouso. Os valores finais de pressão sistólica e diastólica ( $\mathrm{em} \mathrm{mmHg}$ ) foram a média aritmética das duas últimas medidas.

Com base nos resultados clínico-laboratoriais, os participantes foram alocados para quatro grupos:

Grupo 1: sem SM e sem distúrbios glicêmicos (grupo "controle"). Tais indivíduos poderiam apresentar níveis de pressão arterial, triglicérides e HDL-colesterol normais $(28 \%)$ ou apenas um deles anormal $(22,5 \%$ com circunferência da cintura aumentada e hipertensão, $8,1 \%$ com circunferência da cintura aumentada e hipertrigliceridemia e $41,4 \%$ com circunferência da cintura aumentada e HDL-c baixo).

Grupo 2: sem SM e com distúrbios glicêmicos (glicemia de jejum alterada [GJA] ou tolerância à glicose diminuída [TGD] ou DM).

Grupo 3: com SM e sem distúrbios glicêmicos.

Grupo 4: com SM e com distúrbios glicêmicos.

O diagnóstico de SM baseou-se nos critérios da IDF (5), sendo o valor de corte da glicemia de jejum para o diagnóstico de GJA aquele proposto pela $A m e-$ rican Diabetes Association (35). Além de circunferência da cintura aumentada $(>90 \mathrm{~cm}$ para homens ou $>80 \mathrm{~cm}$ para mulheres - valor recomendado para latinos e orientais) deveria haver mais pelo menos dois dos seguintes componentes: triglicérides $\geq 150 \mathrm{mg} / \mathrm{dL}$; HDL-colesterol $<40 \mathrm{mg} / \mathrm{dL}$ ou $50 \mathrm{mg} / \mathrm{dL}$ (homem ou mulher, respectivamente); pressão arterial sistólica $\geq 130 \mathrm{mmHg}$ ou diastólica $\geq 85 \mathrm{mmHg}$ ou uso de antihipertensivos; glicemia de jejum $\geq 100 \mathrm{mg} / \mathrm{dL}$ ou de 2 horas pós-sobrecarga $\geq 140 \mathrm{mg} / \mathrm{dL}$ ou uso de antidiabéticos.

A resistência à insulina e função de célula beta foram avaliadas, respectivamente, pelo HOMA-IR (bomeostasis model assessment for insulin resistance) e o HOMA- $\beta$ (36).

\section{Métodos analíticos}

As dosagens bioquímicas foram realizadas no laboratório do Centro de Saúde da FSP-USP. A glicose plasmática foi determinada pela glicose-oxidase. As concentrações de colesterol total, HDL-c e triglicérides foram determinadas por métodos colorimétricos enzimáticos, processados em analisador automático. As concentrações de LDL-c e VLDL-c foram obtidas por diferença, utilizando-se a equação de Friedewald. Alíquotas de soro foram estocadas a $-80^{\circ} \mathrm{C}$ para posterior determinação de insulina por ensaio imunofluorimétrico, baseado em anticorpo monoclonal (AutoDelfia, Perkin Elmer Life Sciences Inc., Norton, OH, USA) e PCR (ultrassensível) por ensaio imunoenzimático quimioluminescente (Diagnostic Products Corporation, Los Angeles, CA, USA). As determinações de PCR não foram realizadas para indivíduos do grupo 1 (controle). 


\section{Análise estatística}

As variáveis peso, estatura, IMC, CC, RCQ, RCA, massa magra, massa gorda, bioquímicas e HOMA dos grupos 1, 2, 3 e 4 foram expressas como média e desvio-padrão. Aquelas sem distribuição normal sofreram transformação logarítmica (triglicérides e HOMA) para análise. Análise de variância (ANOVA) foi empregada para comparação entre os grupos. O coeficiente de Pearson foi empregado para avaliar a correlação entre as variáveis. O nível de significância foi fixado em $5 \%$. Procedeu-se a análise estatística por meio do programa SPSS 12.0 (SPSS Inc. Woking, Surrey, UK).

\section{RESULTADOS}

Entre os 454 indivíduos incluídos, a maioria era do sexo feminino $(65,8 \%)$, sendo $54 \%$ autoclassificados como brancos, $35,2 \%$ como pardos, $7,5 \%$ como negros e $3,3 \%$ como asiáticos. Foram encontrados 187 participantes sem SM, tendo sido alocados 155 para o grupo l e apenas 32 para o grupo 2 (com distúrbio glicêmico, sendo $78,1 \%$ de GJA e $21,9 \%$ de TGD); dos 267 portadores de SM, 104 apresentavam TOTG normal (grupo 3) e 163, distúrbios glicêmicos (grupo 4). A tabela 1 mostra os dados demográficos, antropométricos e clínicos dos participantes. Participantes do grupo 1 eram mais jovens e tinham, em média, menores valores médios de pressão arterial que os demais. Os grupos 3 e 4 apre- sentaram perfil antropométrico mais desfavorável que aqueles sem SM. A estatura foi similar entre os grupos.

A tabela 2 contém os resultados de variáveis bioquímicas e índice de resistência à insulina dos grupos de participantes. Por definição, as médias dos valores glicêmicos de jejum e pós-sobrecarga foram maiores nos grupos 2 e 4 quando comparados ao 1 e 3 . O perfil lipídico foi pior entre os participantes com SM e DM; mesmo entre aqueles sem SM, as médias das variáveis lipídicas foram ligeiramente mais desfavoráveis que naqueles sem anormalidade glicídica e sem SM. Os grupos com SM tiveram maiores valores médios de insulina e de HOMA-IR, sendo este último mais elevado naquele subgrupo com distúrbio glicêmico. O grupo 2, por sua vez, apresentou o menor valor de HOMA- $\beta$. Comparando-se os grupos 3 e 4, novamente aquele subgrupo com distúrbio glicêmico apresentou média significantemente menor que aquele sem o distúrbio.

Valores de PCR > 1,0 mg/dL foram excluídos, interpretados como indicativos de estado inflamatório clínico e não mais de inflamação subclínica. Diante disso, o número de resultados de PCR especialmente do grupo 1 foi comprometido, não sendo comparado com os demais grupos (Tabela 2).

A totalidade da amostra foi empregada nas análises de correlação para avaliar como as variáveis antropométricas se correlacionavam às metabólicas, aos HOMAs e PCR. Os coeficientes estão apresentados na tabela 3.

Tabela 1. Características clínicas de indivíduos portadores ou não de síndrome metabólica (SM), estratificados segundo a presença (Grupos 2 e 4) ou não (Grupos 1 e 3) de distúrbios glicêmicos

\begin{tabular}{|c|c|c|c|c|c|c|c|c|c|c|c|c|c|}
\hline & \multicolumn{3}{|c|}{$\begin{array}{l}\text { Grupo } 1 \\
\mathrm{~N}=155\end{array}$} & \multicolumn{3}{|c|}{$\begin{array}{l}\text { Grupo } 2 \\
\mathrm{~N}=32\end{array}$} & \multicolumn{3}{|c|}{$\begin{array}{l}\text { Grupo } 3 \\
\mathrm{~N}=104\end{array}$} & \multicolumn{3}{|c|}{$\begin{array}{l}\text { Grupo } 4 \\
\mathrm{~N}=163\end{array}$} & Valor de $p$ \\
\hline Homens (\%) & \multicolumn{3}{|c|}{35,5} & \multicolumn{3}{|c|}{53,1} & \multicolumn{3}{|c|}{31,7} & \multicolumn{3}{|c|}{33,7} & 0,16 \\
\hline Não brancos (\%) & \multicolumn{3}{|c|}{51,6} & \multicolumn{3}{|c|}{40,6} & \multicolumn{3}{|c|}{50,9} & \multicolumn{3}{|c|}{53,4} & 0,58 \\
\hline Idade (anos) & 43,8 & \pm & $14,5^{\mathrm{a}}$ & 51,8 & \pm & $16,3^{b}$ & 56,0 & \pm & $14,3^{c b}$ & 56,1 & \pm & $11,9^{\mathrm{dbc}}$ & $<0,001$ \\
\hline PA sistólica (mmHg) & 122,1 & \pm & $16,5^{\mathrm{a}}$ & 127,8 & \pm & $16,5^{b}$ & 138,1 & \pm & $18,2^{c}$ & 136,9 & \pm & $19,7^{\mathrm{dbc}}$ & $<0,001$ \\
\hline PA diastólica (mmHg) & 76,2 & \pm & $10,3^{\mathrm{a}}$ & 77,9 & \pm & $10,4^{\text {ba }}$ & 83,4 & \pm & $10,7^{c}$ & 81,9 & \pm & $10,1^{d b c}$ & $<0,001$ \\
\hline Peso (kg) & 71,7 & \pm & $15,4^{\mathrm{a}}$ & 66,8 & \pm & $13,4^{\text {ba }}$ & 81,8 & \pm & $18,3^{c}$ & 81,7 & \pm & $14,3^{\mathrm{dc}}$ & $<0,001$ \\
\hline IMC $\left(\mathrm{kg} / \mathrm{m}^{2}\right)$ & 26,9 & \pm & $5,1^{\text {a }}$ & 25,2 & \pm & $4,8^{\text {ba }}$ & 31,2 & \pm & $6,2^{c}$ & 31,3 & \pm & $5,2^{d c}$ & $<0,001$ \\
\hline Circunferência da cintura (cm) & 91,6 & \pm & $13,8^{\mathrm{a}}$ & 87,3 & \pm & $11,5^{\text {bа }}$ & 102,7 & \pm & $13,8^{c}$ & 102,9 & \pm & $10, g^{d c}$ & $<0,001$ \\
\hline Circunferência do quadril (cm) & 102,6 & \pm & $11,4^{\mathrm{a}}$ & 97,6 & \pm & $9,3^{\mathrm{a}}$ & 108,2 & \pm & $12,9^{b}$ & 108,3 & \pm & $11,3^{b}$ & $<0,001$ \\
\hline Razão cintura-quadril & 0,9 & \pm & $0,1^{\mathrm{a}}$ & 0,9 & \pm & $0,1^{\text {ba }}$ & 0,9 & \pm & $0,1^{c}$ & 0,9 & \pm & $0,1^{\mathrm{dc}}$ & $<0,001$ \\
\hline Razão cintura-altura & 56,1 & \pm & $7,9^{\mathrm{a}}$ & 53,8 & \pm & $8,1^{\text {ba }}$ & 63,6 & \pm & $8,5^{c}$ & 63,8 & \pm & $6,8^{\mathrm{dc}}$ & $<0,001$ \\
\hline Massa gorda (\%) & 31,1 & \pm & $10,3^{\mathrm{a}}$ & 25,3 & \pm & $8,8^{b}$ & 35,4 & \pm & $8,5^{c}$ & 34,9 & \pm & $8,6^{\mathrm{dc}}$ & $<0,001$ \\
\hline
\end{tabular}

Dados expressos em porcentagem (comparados pelo qui-quadrado) ou média e desvio-padrão (comparados por ANOVA).

Letras diferentes indicam diferença significante.

IMC: índice de massa corpórea; PA: pressão arterial. 
Tabela 2. Valores médios de variáveis bioquímicas e índice de resistência à insulina dos 4 grupos de participantes, estratificados segundo a presença ou não de síndrome metabólica e de distúrbios glicêmicos

\begin{tabular}{|c|c|c|c|c|c|c|c|c|c|c|c|c|c|}
\hline \multirow[b]{2}{*}{ Glicemia jejum (mg/dL) } & \multicolumn{3}{|c|}{ Grupo 1} & \multicolumn{3}{|c|}{ Grupo 2} & \multicolumn{3}{|c|}{ Grupo 3} & \multicolumn{3}{|c|}{ Grupo 4} & \multirow{2}{*}{$\begin{array}{c}\text { Valor de } \mathbf{p} \\
<0,001\end{array}$} \\
\hline & 86,77 & \pm & $8,06^{\mathrm{a}}$ & 109,13 & \pm & $17,59^{b}$ & 88,30 & \pm & 7,39са & 113,27 & \pm & $22,84^{\mathrm{db}}$ & \\
\hline Glicemia 2h (mg/dL) & 96,24 & \pm & $17,85^{\mathrm{a}}$ & 121,59 & \pm & $54,06^{\mathrm{b}}$ & 102,39 & \pm & $19,16^{\text {ca }}$ & 148,69 & \pm & $54,69^{\mathrm{db}}$ & $<0,001$ \\
\hline Triglicérides (mg/dL) & 98,19 & \pm & $53,77^{\mathrm{a}}$ & 112,13 & \pm & $47,10^{\text {ba }}$ & 170,40 & \pm & $72,25^{c}$ & 155,01 & \pm & $90,75^{d c}$ & $<0,001$ \\
\hline Colesterol total (mg/dL) & 180,19 & \pm & $38,91^{\mathrm{a}}$ & 190,84 & \pm & $40,13^{\text {ba }}$ & 204,46 & \pm & $41,95^{\mathrm{cb}}$ & 197,16 & \pm & $42,70^{\mathrm{dbc}}$ & $<0,001$ \\
\hline LDL-c (mg/dL) & 113,10 & \pm & $35,11^{\mathrm{a}}$ & 118,53 & \pm & $35,72^{\text {ba }}$ & 129,98 & \pm & $37,17^{c b}$ & 124,11 & \pm & $38,93^{\mathrm{dbc}}$ & $<0,001$ \\
\hline HDL-c (mg/dL) & 47,55 & \pm & $12,42^{\mathrm{a}}$ & 48,25 & \pm & $13,76^{\text {ba }}$ & 39,89 & \pm & $8,51^{c}$ & 42,22 & \pm & $11,33^{d c}$ & $<0,001$ \\
\hline Insulina de jejum (uUI/ml) & 6,10 & \pm & $5,08^{\mathrm{a}}$ & 6,47 & \pm & $4,03^{\text {ba }}$ & 9,71 & \pm & $6,44^{c}$ & 12,75 & \pm & $15,34^{d c}$ & $<0,001$ \\
\hline PCR (mg/dL) & & ND & & 0,24 & \pm & 0,23 & 0,31 & \pm & 0,25 & 0,34 & \pm & 0,24 & 0,41 \\
\hline HOMA-IR & 1,33 & \pm & $1,25^{\mathrm{a}}$ & 1,67 & \pm & $1,00^{\text {ba }}$ & 2,12 & \pm & $1,40^{c b}$ & 3,35 & \pm & $4,06^{d}$ & $<0,001$ \\
\hline HOMA- $\beta$ & 104,40 & \pm & $81,14^{a}$ & 58,56 & \pm & $43,77^{b}$ & 156,34 & \pm & $131,75^{c}$ & 109,00 & \pm & $127,94^{\text {da }}$ & $<0,001$ \\
\hline
\end{tabular}

Dados expressos como média e desvio-padrão, comparados pela ANOVA.

Letras diferentes indicam diferença significante.

ND: não disponível.

HOMA: homeostasis model assessment, PCR: proteína $\mathrm{C}$ reativa.

Tabela 3. Coeficientes de correlação entre variáveis antropométricas e metabólicas da totalidade dos participantes

\begin{tabular}{|c|c|c|c|c|c|c|c|c|c|}
\hline & $\begin{array}{c}\text { Glicemia de } \\
\text { jejum }\end{array}$ & $\begin{array}{c}\text { Glicemia de } \\
2 h\end{array}$ & Triglicérides & LDL & HDL & Insulina & PCR & HOMA-IR & НОМА- $\beta$ \\
\hline IMC & $0,132^{* *}$ & $0,150^{* *}$ & $0,236^{* *}$ & $0,095^{*}$ & $-0,086$ & $0,501^{* *}$ & $0,285^{* *}$ & $0,499^{* *}$ & $0,378^{\star *}$ \\
\hline CA & $0,170^{* *}$ & $0,171^{* *}$ & $0,311^{* *}$ & $0,119^{*}$ & $-0,143^{* *}$ & $0,493^{* *}$ & $0,280^{* *}$ & $0,503^{* *}$ & $0,335^{* *}$ \\
\hline$C Q$ & 0,076 & 0,119 & $0,114^{*}$ & 0,090 & $-0,010$ & $0,426^{* *}$ & $0,218^{* *}$ & $0,416^{* *}$ & $0,350^{* *}$ \\
\hline $\mathrm{RCQ}$ & $0,196^{* *}$ & $0,145^{*}$ & $0,384^{* *}$ & 0,085 & $-0,226^{* *}$ & $0,258^{* *}$ & 0,145 & $0,290^{* *}$ & 0,082 \\
\hline RCA & $0,160^{*}$ & $0,189^{* *}$ & $0,289^{* *}$ & $0,172^{\text {** }}$ & $-0,065$ & $0,506^{* *}$ & $0,317^{\star \star}$ & $0,513^{* *}$ & $0,351^{* *}$ \\
\hline$M G$ & 0,054 & $0,118^{*}$ & 0,087 & $0,110^{*}$ & $0,154^{*}$ & $0,352^{* *}$ & $0,233^{* *}$ & $0,336^{* *}$ & $0,314^{* *}$ \\
\hline
\end{tabular}

IMC: índice de massa corpórea; CC: circunferência da cintura; CQ: circunferência do quadril; RCQ: razão cintura-quadril; RCA: razão cintura-altura; PCR: proteína C reativa.

${ }^{*} \mathrm{P}<0,05 ;{ }^{* *} \mathrm{P}<0,01$

Em decorrência do baixo número e das características clínicas dos indivíduos que compuseram o grupo 2, essa análise também foi realizada excluindo esses participantes, não tendo sido observada qualquer alteração na significância achados de correlação. Correlações fracas, porém significantes, foram observadas de certas medidas antropométricas (IMC, circunferência da cintura, RCQ e RCA) com as glicemias e triglicérides. A circunferência da cintura e a RCQ se correlacionaram negativamente com a HDL-c. Foram encontradas correlações moderadas da antropometria com a PCR, sendo o coeficiente da RCA o mais elevado $(\mathrm{r}=0,317$; $\mathrm{p}<0,01)$. Também os índices de resistência à insulina - insulinemia de jejum e HOMA-IR - apresentaram boas correlações com medidas antropométricas; novamente, a RCA apresentou os coeficientes mais elevados $(\mathrm{r}=0,506$ e 0,513 , respectivamente para insulinemia $\mathrm{e}$ HOMA-IR, $\mathrm{p}<0,01)$. Em análise com exclusão dos 32 indivíduos do grupo 2, os coeficientes foram semelhantes e mantiveram a mesma significância estatística (dados não mostrados). $O$ HOMA- $\beta$ não se correlacionou significantemente apenas com a RCQ.

\section{DISCUSSÃO}

Os achados do presente estudo revelam que, em amostra da população brasileira, qualquer das medidas antropométricas analisadas identifica indivíduos com SM, mas não parece capaz de diferenciar aqueles com distúrbios glicêmicos. Não apoiam a hipótese de que a presença de anormalidades do metabolismo glicídico per se estaria associada a pior perfil antropométrico, especialmente no que se refere à deposição abdominal de gordura. A antropometria dos grupos de indivíduos com distúrbios glicêmicos ( 2 e 4 ), aleatoriamente selecionados na rede pública de saúde, foi semelhante à dos 
grupos sem tais anormalidades ( 1 e 3). Por outro lado, tais achados reforçaram a relação mais forte de medidas de adiposidade central com resistência à insulina, sugerindo inclusive utilidade da razão cintura-altura.

Para todas as variáveis antropométricas, diferenças marcantes ocorreram quando se considerou a presença (grupos 3 e 4) ou ausência (l e 2) de SM. Os indivíduos dos grupos sem SM apresentavam-se, em média, na faixa de sobrepeso (IMC entre 25 e $30 \mathrm{~kg} / \mathrm{m}^{2}$ ), enquanto aqueles com SM, na faixa de obesidade (IMC $\geq 30 \mathrm{~kg} / \mathrm{m}^{2}$ ). Ambos os grupos com SM apresentaram médias de valores antropométricos significantemente maiores, e ter ou não anormalidades glicêmicas não contribuiu para diferenciar os subestratos neste estudo. Porém, não podemos afastar que diferenças no nível de adiposidade intra-abdominal existam entre indivíduos sem e com anormalidades glicêmicas e que só seriam detectadas apenas por meio de métodos altamente especializados e sensíveis, como é o caso da tomografia computadorizada, ou com amostras maiores.

Entre os participantes deste estudo, houve predomínio de mulheres; habitualmente essa situação é observada em campanhas com apelos de saúde ou mesmo em ambulatórios da rede pública de saúde. A diversidade de cores de pele dos indivíduos incluídos expressa a miscigenação e heterogeneidade étnica de nossa população, fato que era desejável para a presente análise. Deve-se ressaltar que analisamos uma amostra de conveniência, portanto não representativa da nossa população, mas que mantiveram proporções de não brancos similares nos subgrupos estudados. Considerando o Brasil de hoje, há predomínio de indivíduos não brancos, enquanto nesta amostra do Município de São Paulo constatou-se percentual de $46 \%$.

Tendo em mente o conceito de SM, era esperado que os indivíduos portadores da SM tivessem pior perfil cardiometabólico que os grupos sem a SM. Porém, desconhecíamos quais seriam as características do grupo sem a SM que desenvolveram distúrbio glicêmico. $\mathrm{O}$ encontro de valores antropométricos, pressóricos e bioquímicos semelhantes entre não portadores de $S M$ com e sem anormalidade da tolerância à glicose sugere que, pelo menos em parte, esses indivíduos não apresentem o DM tipo 2 clássico. Chamou à atenção o reduzido tamanho da amostra do grupo 2 (sem SM e com distúrbio glicêmico), bem como o grau e a distribuição da adiposidade comparáveis aos do grupo l. É possível que participantes do grupo 2 não desenvolvam o clássico DM tipo 2 associado à obesidade. A não disponibi- lidade de parâmetros mais específicos para classificação do tipo de DM (como a determinação de anticorpos anti-insulina e anti-GAD) não permite avançar na especulação de que esse grupo poderia estar "contaminado" com indivíduos portadores de lesão pancreática autoimune, compatível com DM tipo 1. Outro achado do presente estudo que reforça a última hipótese foi a constatação de que o mesmo grupo 2 apresentou a menor média de HOMA- $\beta$, indicando menor capacidade secretória de insulina, coerente com destruição autoimune das células beta.

As correlações encontradas foram compatíveis com o esperado, uma vez que é amplamente conhecido que o aumento da adiposidade corporal induz anormalidades metabólicas, em especial aquela de distribuição central $(4,22,26)$. De fato, o IMC, a circunferência da cintura, a RCQ e a RCA se correlacionaram com as concentrações de glicose e triglicérides. Havia a expectativa de que a RCA pudesse revelar coeficientes mais fortes que outras medidas antropométricas $(29,30)$, sinalizando mais eficazmente indivíduos brasileiros com distúrbios metabólicos. Seria interesse dispor de uma medida reprodutível e de baixo custo que pudesse ser usada amplamente numa população com tamanha heterogeneidade étnica.

De interesse foi o fato de a RCQ ter apresentado o mais alto coeficiente de correlação com as concentrações de triglicérides, critério fundamental para diagnóstico da SM. Esse achado reforça a opinião de outros pesquisadores sobre a importância da "cintura hipertrigliceridêmica" na predição de risco cardiovascular $(37,38)$. Não podemos afastar que a RCA pudesse apresentar melhores correlações em amostras maiores $\mathrm{e}$ mais representativas da nossa população. De qualquer forma, a natureza transversal deste estudo limitaria inferir sobre relações tipo causa-efeito entre as variáveis analisadas.

Os achados de correlações da insulinemia de jejum e do HOMA-IR com medidas antropométricas foram consistentes e coerentes com o esperado, uma vez que é sabido que a adiposidade corporal deteriora a sensibilidade à insulina (6,7). Mesmo o IMC - que fornece estimativa da gordura generalizada e não central - apresentou boas correlações com parâmetros de resistência à insulina. $\mathrm{O}$ mais interessante foi o encontro de correlação da RCA com a PCR e índices de resistência à insulina (insulinemia de jejum e HOMA-IR), de magnitude moderada, sugerindo que esse indicador antropométrico pode ser útil para revelar o estado pró-inflamatório 
e de resistência à insulina. Apesar de o coeficiente de correlação da RCA ter sido o mais alto entre as variáveis antropométricas aqui estudadas, não é possível afirmar que a força das correlações seja diferente entre si.

Em concordância com outros estudos que encontraram valores de PCR associados diretamente com medidas de adiposidade (8), a RCA apresentou o mais alto coeficiente de correlação se comparado a outras medidas. Tal encontro é compatível com a hipótese de que o tecido adiposo predispõe a um estado pró-inflamatório devido à produção de adipocitocinas (39). Interleucinas secretadas especialmente no tecido adiposo visceral estimulam a síntese hepática de proteínas de fase aguda, sendo a PCR a mais comumente empregada para completar a avaliação do risco cardiovascular (10).

No que diz respeito às correlações diretas e significantes também com o HOMA- $\beta$, poderiam sugerir que as células $\beta$ estão sendo requisitadas para secretar mais insulina à medida que aumenta a adiposidade corporal; porém, a transversalidade deste estudo não permite inferir tal relação causal.

Em suma, em amostra da população brasileira residente em São Paulo, nossos achados indicam que qualquer das medidas antropométricas analisadas identifica indivíduos com SM, mas não parece capaz de diferenciar aqueles com distúrbio glicêmico. Reforçamos a relação mais forte das medidas de adiposidade central com resistência à insulina, sugerindo utilidade para a RCA. É possível que componente autoimune possa ter contribuído para o comprometimento do metabolismo glicídico dos participantes do grupo 2. Mais estudos envolvendo amostras representativas e de delineamento longitudinal são necessários para comprovar tais proposições.

Agradecimentos: Este trabalho contou com auxílio à pesquisa $\mathrm{e}$ bolsa de estudo da Fundação de Amparo à Pesquisa do Estado de São Paulo (Fapesp).

Declaração: os autores declaram não haver conflitos de interesse científico neste estudo.

\section{REFERÊNCIAS}

1. World Health Organization. Preventing and managing the global epidemic. Report of a WHO consultation on obesity. Geneva: World Health Organization (WHO/NUT/NDC/98.1); 1998.

2. Monteiro CA, Mondini L, de Souza AL, Popkin BM. The nutrition transition in Brazil. Eur J Clin Nutr. 1995; 49:105-13.

3. Flegal KM, Carroll MD, Kuczmarski RJ, Johnson CL. Overweight and obesity in the United States: prevalence and trends, 19601994. Int J Obes. 1998;22:39-47.
4. Depres JP, Moorjani S, Lupien PJ, Tremblay A, Nadeau A, Bouchard C. Regional distribution of fat, plasma lipoproteins, and cardiovascular disease. Arteriosclerosis. 1990;10:497-511.

5. Alberti KGMM, Zimmett P, Shaw J. Metabolic syndrome-a new world-wide definition. A consensus statement from the International Diabetes Federation. Diabetic Medicine. 2006;23:469-80.

6. Reaven GM. Role of insulin resistance in human disease. Diabetes. 1988;37(12):1595-607.

7. Reaven GM. Role of insulin resistance in human disease (syndrome X): an expanded definition. Annu Rev Med. 1993;44:121-31.

8. Ridker PM, Wilson PW, Grundy SM. Should C-reactive protein be added to metabolic syndrome and to assessment of global cardiovascular risk? Circulation. 2004;109:2818-25.

9. Visser M, Bouter LM, McQuillan GM, et al. Elevated C-reactive protein levels in overweight and obese adults. JAMA. 1999;282:2131-5.

10. Ridker PM, Rifai N, Rose L, et al. Comparison of C-reactive protein and low-density lipoprotein cholesterol levels in the prediction of first cardiovascular events. N Engl J Med. 2002;347:1557-65.

11. Lorenzo C, Williams K, Hunt KJ, Haffner SM. The metabolic syndrome as predictor of type 2 diabetes: the San Antonio Heart Study. Diabetes Care. 2003;26(3):153-9.

12. Ford ES, Schulze MB, Pischon T, Bergmann MM, Joost HG, Boeing H. Metabolic syndrome and risk of incident diabetes: findings from the European Prospective Investigation into Cancer and Nutrition-Postdam Study. Cardiovasc Diabetol. 2008;7:35.

13. Lakka HM, Laaksonen DE, Lakka TA, Niskanen LK, Kumpusalo $\mathrm{E}$, Tuomilehto $\mathrm{J}$, et al. The metabolic syndrome and total and cardiovascular disease mortality in middle-aged men. JAMA. 2002;288:2709-16.

14. Malik S, Wong ND, Franklin SS, Kamath TV, L'Italien GJ, Pio JR, et al. Impact of the metabolic syndrome on mortality from coronary heart disease, cardiovascular disease, and all causes in United States adults. Circulation. 2004;110:1245-50.

15. Fontbonne A, Charles MA, Thibult N, Richard JL, Claude JR, Warnet JM, et al. Hyperinsulinemia as a predictor of coronary heart disease mortality in a healthy population: the Paris Prospective Study, 15-year follow-up. Diabetologia. 1991;34:356-61.

16. Haffner SM, Lehto S, RönnemaaT, Pyörälä K, Laakso M. Mortality from coronary heart disease in subjects with type 2 diabetes and in nondiabetic subjects with and without prior myocardial infarction. NEJM. 1998;339:229-34.

17. Alexander CM, Landsman PB, Teutsch SM, Haffner SM. NCEPdefined metabolic syndrome, diabetes, and prevalence of coronary heart disease among NHANES III participants aged 50 years and older. Diabetes. 2003;52:1210-4.

18. Coutinho M, Gerstein HC, Wang Y, Yusuf S. The relationship between glucose and incident cardiovascular events. A metaregression analysis of published data from 20 studies of 95,783 individuals followed for 12.4 years. Diabetes Care. 1999;22:233-40.

19. Haffner SM. Insulin resistance, inflammation, and the prediabetic state. Am J Cardiol. 2003;92(4A):18J-26J.

20. Stevens J, Keil JE, Rust PF, Verdugo RR, Davis CE, Tyroler HA, et al. Body mass index and body girths as predictors of mortality in black and white men. Am J Epidemiol. 1992;135:1137-46.

21. Prospective Studies Collaboration. Body-mass index and causespecific mortality in 900000 adults: collaborative analyses of 57 prospective studies. Lancet. 2009;373:1083-96.

22. Han TS, Van Leer EM, Seidell JC, Lean ME. Waist circumference action levels in the identification of cardiovascular risk factors: prevalence study in a random sample. Br Med J. 1995;311:1401-5.

23. Pischon $T$, Boeing $H$, Hoffmann $K$, Bergmann $M$, Schulze MB, Overvad K, et al. General and abdominal adiposity and risk of death in Europe. N Engl J Med. 2008;359:2105-20. 
24. Despres JP, Lemieux I, Bergeron J, Pibarot P, Mathieu P, Larose E, et al. Abdominal obesity and the metabolic syndrome: contribution to global cardiometabolic risk. ArteriosclerThrombVasc Biol. 2008;28:1039-49.

25. Alberti KGMM, Zimmett P, Shaw J. Metabolic syndrome-a new world-wide definition. A consensus statement from the International Diabetes Federation. Diabetic Medicine. 2006;23:469-80.

26. Krotkiewski M, Bjorntorp P, Sjostrom L, Smith U. Impact of obesity on the metabolism in men and women: importance of regional adipose tissue distribution. J Clin Invest. 1983;72:1150-62.

27. Hsieh SD, MutoT. Metabolic syndrome in Japanese men and women with special 309 references to the anthropometric criteria for the assessment of obesity: proposal to use the waist-to-height ratio. Prev Med. 2006;42:135-9.

28. Hsieh SD, MutoT.The superiority of waist-to-height ratio as an anthropometric index to evaluate clustering of coronary risk factors among non-obese men and women. Prev Med. 2005;40:216-20.

29. Mombelli G, Zanaboni AM, Gaito S, Sitori CR. Waist-to-height is a highly sensitive index for the metabolic syndrome in a Mediterranean population. Metab Synd Relat Disord. 2009;7(5):477-84.

30. MacKay MF, Haffner SM, Wagenknecht LE, D’Agostino RB Jr, Hanley AJ. Prediction of type 2 diabetes using alternate anthropometric measures in a multi-ethnic cohort: the insulin resistance atherosclerosis study. Diabetes Care. 2009;32(5):956-8.

31. Ribeiro-Filho FF, Faria AN, Azjen S, Zanella MT, Ferreira SRG. Methods of estimation of visceral fat: advantages of ultrasonography. Obes Res. 2003;11(12):1488-94.
32. IBGE - Instituto Brasileiro de Geografia e Estatística. Estudo Nacional das Despesas Familiares - ENDEF: 1974-1975. Rio de Janeiro, RJ; 1978.

33. World Health Organization. Diet, nutrition and the prevention of chronic diseases. Report of a WHO group. Technical Report Series No 797. World Health Organization, Geneva; 1990.

34. Garnett SP, Baur LA, Cowell CT. Waist-to-height: a simple option for determining excess central adiposity in young people. Int $\mathrm{J}$ Obes. 2008;32:1028-30.

35. American Diabetes Association. Diagnosis and classification of diabetes mellitus. Diabetes Care. 2008;31(S1):S55-60.

36. Matthews DR, Hosker JP, Rudenski AS, Naylor BA, et al. Homeostasis model assessment: insulin resistance and B-cell function from fasting plasma glucose and insulin concentrations in man. Diabetologia. 1985;28:412-19.

37. Lemieux I, Pascot A, Couillard C, Lamarche B, et al. Hypertriglyceridemic waist: a marker of the atherogenic metabolic triad (hyperinsulinemia; hyperapolipoprotein B; small, dense LDL) in men? Circulation. 2000;102:179-84.

38. Kahn HS, Valdez R. Metabolic risks identified by combination of enlarged waist and elevated triacylglycerol concentration [see comment]. Am J Clin Nutr. 2003;78:928-34.

39. Bulcão C, Ferreira SRG, Giuffrida FMA, Ribeiro-Filho FF. The new adipose tissue and adipocytokynes. Curr Diabetes Rev. 2006;2(1):19-28. 